\title{
Socio-Cultural Values and Ecological Awareness in Sakela Sili in Kirant Rai in Nepal
}

Prakash Rai

Assistant Professor of English,

Bhojpur Multiple Campus, Bhojpur, Nepal.

https://doi.org/10.3126/litstud.v34i01.39537

\section{Abstract}

In the Eastern part of Nepal, Kirant Rai in traditional attires perform Sakela Sili, a dance style performed twice a yearin a larger circle to honour of Sakela, a deity in Kirant Rai community. The performance of Sakela or Sakkew involves singing and dancing simultaneously. Sakela connects the Kirant to their original source of energy, cultural root, origin and the civilization. Ethnic Kirant Rai, including youth and the old in their dance steps of working in the farmland and worshiping gods, with their hands and legs raising low and high, embody their connections to the terrestrial and celestial, profane and sacred, and the humanity and the divinity to maintain a perfect balance of art and life. The dancers in their body movements blend their passionate intensity to work and aesthetic response to art and embody socio -cultural practices and ecological awareness. While dancing, they work and dance for representing the life in totality. The Kirant Rai work pleasingly, and they dance with their strong passion to work. This paper as an instance of qualitative research employs both emic and etic perspectives to find out how such Sakela Sili performed shapes the socio - cultural values and ecological awareness among Kirant Rai community.

Keywords: Sakela Sili, Socio-Cultural Value, Performance, Ethnic Identity, Ecological Awareness

\section{Introduction}

Sakela Sili is a distinct cultural dance based more on acting than stepping performed by the Kirant Rai in the honour of a powerful deity, Sakela, or Sakkhewa, linked with natural forces and ancestral entities. Kirant Rai also entitles to Sakela as Sakkhewa, Chasok and Sakenwa, depending on linguistic, clan and regional variations (Rai 14). In this way, Kirant Rai, living in different geographical locations, have been entitling Sakela as Sakkhewa, Chasok, Chasuwa, Toshi ever since the inception of tradition. The various names for Sakela refer to the essence and magnanimity of nature - stone, soil, water and vegetation. Taking Sakela Sili into account that occupies the most important space of socio-cultural life of Kirant Rai, many scholars and researchers have studied different aspects of Sakela Sili. For example, Kamal Jung Rai has studied history, 
evolution and structural aspects while Dik Bahadur Rai carried out research on impacts of modernization and globalization on Sakela Sili. However, this article aims at exploring how Sakela Sili has been shaping the socio-cultural values and ecological awareness of Kirant Rai in their daily lives and what factors of Sili influence them to conform to the rules and tradition. In other words, drawing an underlying interrelationship between the Sakela Sili and Kirant Rai's socio-cultural behaviors and practices and ecological awareness both at individual and group level is major contention of this paper.

Observed twice a year during Ubhauli, ascending time that falls around June and Udhauli, descending time that falls about December with a joy and utmost importance, Sakela Sili invariably establishes the way Kirant Rai believe in, behave with, and interact with others in their day to day lives.Sakela is the name of the almighty deity Kirant Rai have been worshipping and revering to since their early evolution while Sili is the dance step and style focused more on acting and expressing than just performing, performed to please Sakela simultaneously with worshiping. The procedures for worshipping and dance performance are based on the $M u d d^{l}$, which is the guiding philosophy of all cultural cults, and ritual performances in Kirant Rai community. In this case, Prabin Puma observes Sili practiced and performed during Sakela worship intrinsically adheres to the rules and traditions of the Muddum (44).Scholars on culture and rituals, Nakchhong, main knowledgeable priest with shamanic power and Munddum experts don't have unanimous opinions on the origin, history and tradition of Sakela worship and Sili performance, though folk belief and oral-ritual practice govern the tradition of festive performance. Etymologically, Sakmawa, originally in Rai language, meaning 'water', gets bifurcated into Sakma and wa, which mean 'life' and 'water' respectively (K. Rai, Personal Interview). Water as life-saving force essential for living world is worshipped and revered in the tradition. Shrestha, regarding Sakela worship and Sili performance tradition, states:

Sakela, perormative festival observed by Kirant Rai, was commenced by the first peasant, Hechhakuppa and Muksima to appease Sakela, natural divine force and ancestral deities so that human beings get healthy, happy and prosperous. The stone kept upright position in the altar, standing for Sakela, designates Sisamlung, Chhanmalung, Longmalung, Sakkhewalung is worshipped invoking to protect nursery plants, crops, and agricultural yield from natural disasters. (3)

\footnotetext{
${ }^{1}$ Kirant philosophy in oral form
} 
Given the fact whatShresta puts forward, worshipping Sakela and Sili performance, which was believed to be initiated by the first Kirant peasant, Hechhhakupa, commenced the tradition intending to placate Sakela, the supreme power, mainly linked with nature and ancestral linage for the well-being of humans and agricultural crops and plants. From the fact presented, it proves that the tradition of Sakela Sili began with the advent of the agricultural age, which is mainly held twice a year during Ubhauli, ascending season, which falls in June i. e. Baishakhe Purnima and Udhauli, descending season in December, i. e. Mangsire Purnima though some Sili reflect the life style of haunting age. It is the Sakela Than $^{2}$ wherein Nakchhong ${ }^{3}$ as well as other participants, performers worship and dance with different Sili by conforming to the tradition. Nonetheless, Sakela Sili at present takes place both in the Than, original altar, in the village and in the artificial altar in the urban cities like Tudikhel, open space for army parade.

Constituting human and non-human elements, without which the performance is impossible, Sakela Sili is essentially performed with leadership of Nakchhong, main priest with shamanic power, who mediates in between the deity, Sakela and ordinary people. In order to assist Nakchhong, Silihangpa, main male choreographer, Silihangma, main female choreographer for directing Sili movements in the performance and ordinary dance performers and dancers including youths, elders, men, women from Kirant Rai and non- Kirant Rai play important roles in a due course of dancing and performing Sili. Performed nationally as well as internationally at present context due to intra-national and transnational migration, Sakela Sili draws wide attention not only in Kirant Rai community but also non-Kirant Rai community because people from all walks of life living in adjacent to Kirant community participate in dance performance. The synchronous and tradition-bound dance performance by all means frames the socio-cultural and ecological perspectives of Kirant Rai not because they mimic daily life activities but because they internalize the values the Sili inherently inculcates into the heart and minds of the people.

\section{Methodology}

The paper has majorly been based on qualitative research method, employing simultaneously the primary and secondary sources of data collected and analysed through emic and etic perspectives as well as ethnographic inquiry. Since I belong to Kirant Rai community, I have

\footnotetext{
${ }^{2}$ Original altar, constructed by the divine power of the Nakchhong

${ }^{3}$ The major priest with shamanic/divine power in Kirant community
} 
observed and participated in worshipping and performing Sakela Sili ever since I knew it was a great festival of Kirant Rai. My insider and outsider point of view on it at the same time provided me with a sort of insights and linkage to find out the relationship between the Sili and their reflections on socio-cultural lives of Kirant Rai. The in-depth interview with ritual experts, including Nakchhong, Assistant Nakchhong, knowledgeable seniors and Kirant Rai academicians, Focused Group Discussion, observation and ethnographic narration have minutely been used as principal tools for collecting data and interpreting as well as analyzing for the results that unveiled the socio- cultural and ecological implications of Sakela Sili in the Kirant Rai that they intrinsically as well as extrinsically reflect on social discourse and social practice. The texts and materials related to Sakela Sili such as Sakkhwa Sili: Ek Adhyan, a study, by Kamal Jung Rai, The impacts of Modernization on the Traditional Sakewa Festival in the Rai Kirat Community of Nepal: A Case Study of the Rai Community by Dik Bahadur Rai etcetera function as the secondary sources for the study. For the interpretive theoretical frameworks, I have exploited the magical perspective and practice of Luc Sala, socio-cultural ideas of Victor Turner, the concept of Ecosophy or Deep Ecology of Arne Naess, and aesthetic beauty and metaphorical representation of Dodd Sheril.

\section{Sakela Sili and Socio-Cultural Values}

Sakela in Kirant Rai is socio-culturally very important because it has been predominantly shaping the patterns of socio-cultural behavior, practices and values. Considering Sakela as one of the greatest cultural assets in Kirant Rai community, B. Rai states, "Sakela has utmost paramount space in making and remaking of Kirant identity formation process that each and every ethnic indigenous group aspired to form for identity and empowerment" (Personal Interview). It means performingSakela on diverse themes and activities of life connects symbolic interactions to real life experience and dynamisms. With regards to types of Sakla Sili that Kirant Rai practice and adopt in the performance, R. Rai, Nakchhong from Dilpa Nagi maintains:

A variety of Sakela Sili has popularly been practiced and performed representing life, ranging from birth to death, love to hate, primitive to modern, haunting to agriculture etcetera since its inception. Among them, Paruhang-Sumdi Sili, and Aaptarung Sili are the notable Sili under which other sillies exist for performance. KharuSili performance based on early initial phase of agriculture age, including works of clearing off the fertile land, burning the dried plants and shrubs, making digging tools out of woods, 
preparing land by mixing ashes and soil, sowing seeds etcetera showcases the preliminary era of agriculture age of human civilization. He adds, "the tradition of agricultural works, vividly shown in the dance performance still prevails not only in Kirant community but also other nonKirant communities in Nepal." (Personal Interview)

Shaping the practice of agricultural evolution among Kirant Rai communities, Kharu Sili, one of popular and frequently performed Sili, by and large, seems to be contributing to the development of socioagricultural aspects that Kirant Rai are still adopting in their lives even today. Kirant Rai populations mostly based on rural setting in Nepal even at present depend on agriculture, primarily, paddy, maize and millet yields. Worshipping Sakela, deity, a symbol of nature and ancestral spirit is meant to revere and maintain the hierarchical system of our social values. The bodily movement during the dance performance portrays the whole picture of human experiences and social activities.

In addition, "Chhekusonma Sil, bases on the courtship practice in between a young boy and a young girl, shows romantic courtship tradition in Kirant Rai community"(K. Rai 184). In this dance, love affair that actually takes place in real life is portrayed and vice versa. On account of this dance tradition of romantic love affairs, Kirant Rai adheres to love marriage even at present time. Regarding human body and experience, Turner pointed out that human bodily experiences (the 'organic') are the main origin of cultural classifications and symbolic meaning (the 'superorganic'), but he also demonstrated that individual experience and body are inseparable from relations between people and their shared stock of cultural knowledge, and that it is only within these socio-cultural structures that bodily experiences become meaningful (89-91). The bodily movement in the Sili performance closely showcases the canvass of experiences - bitter and sweet, imagined and real, love and hate, comic and tragic - the ways Kirant Rai have been embracing in their lives.

The relationship between the human body and specific practices goes side by side, in which one affects the other. The motivation for eating a particular food item might include beliefs, religious and ritual purposes, as well as the construction of self and body image. The performative on agricultural works shows that relationships are the key to understanding how soils influence people who create and use them. The Kharu Sili influences the way Kirant Rais interact with the world for understanding people and society because their unconscious relationship to their surroundings they work and live by creating and fostering the tradition. 


\section{Social Solidarity}

Social integration and solidarity are invariably based on mutual reciprocity, cooperation, common shared values and rituals as well as cultural performance. About Sakela Sili performance, B. Rai articulates, "Nakchhong plays the role of an intermediary between the worlds of the dead and the living, physical and spiritual, man and God in order to maintain the balance and an equation in the complex artifice of the world," (Personal Interview), wherein people remain attached to one another because of their shared knowledge and common belief. Common cultural practices usually involving prayers, offerings, and sacrifices with as ritual objects and Sili performance contributed to social solidarity and integration. With the ritual performance involving multiple tiers of personalities such as Nakchhong, Silihangpa, Silihangma, and other performers unites humans and non-humans, thereby making them realize their space and recognition in defining their identity and self-respect. As Nakchhong mediates in between seen and unseen, natural and supernatural, each and every Sili performers play their respective roles.

Sili performed to mimic haunting to agricultural works Kirant Rai embraced since their origin plays a central role in the formation of memory, identity community and worldview in the Kirant community. The production/ construction of material artifacts is inclusive of social action: the construction of landscapes and cosmology that material culture involves bringing of symbolic meaning to physical matter, either through physical transformations or the incorporation into symbolisms of social practices which includes cultural landscapes as well as artefacts. Reflecting the different dimension of human life, B. Rai adds:

Fakelewa Sili mimics the union and separation of beloved and dear ones-ranging from exchanging memento to departing each other - to portray the reality of the life. This showcases not only the picturesque of love tragedy at modern time but also poignantdemise of loved ones. The social relationship ranging from love between young boy and girl to love between family members like brother and sister is artistically simulated and reflected on the Sili. The personal, family and social relationship about uniting and separating in different contexts presented beautifully in the Sili persist on socio-cultural lives of Kirant Rai even today. (Personal Interview)

The idea articulated by K. Rai implies that the relationship that ranges from uniting to separating among family members and social members reflected on the dance lays the foundation of social solidarity and integration. The moment of joy and the moment of tears both establish 
the values of sharing and caring that really assist our social system. In this matter, Salisbury reports, "the simultaneous transmission and inscription of the social memory and community as a relational entity (made up among other things) by networks of people and affordances functioning at multiple spatial and temporal scales" (108), intimates that it is the chain of people and their relationship per se with some tradition-bound system that transmit and in script the history, root and memory of a particular community. The spatial and temporal factors that function at multiple tiers appear to be extremely important for systematic operation of social networks and relation that govern our social hierarchy and cultural world. As far as Sakela Sili is concerned, performed twice a year in the formal and informal setting - original altar and artificial altar made for the time being such open space, ground-produces the values of structured social system and strong networks of people with their respective roles for social unity in diversity.

\section{Co-existence and Harmony}

Sakela Sili performance that mainly relies on the directorship as well as mentorship of the Nakchhong signifies developing harmonic relationship and feeling of co-existence among performers. According to B. Rai, "Dancing steps and movements led by Silihangma, female choreographer and Silihangpa, male choreographer, with resonance of musical rhythms and songs emerged out of natural phenomena uniformly, instill the value of obedience, system, hierarchy, and social order in Kirant Rai community to expand the horizon of human universe of art, culture and civilization"(Personal Interview) . Dancing and performing together regardless of age group, social status and rank symbolizes friendship, unity and conviviality connecting the physical world with spiritual world as long as the performers get so much engrossed in dancing that they plunge into the sea of pleasure and meditation. As a performer, while performing in Sili having had similar feeling of engrossing into the deep abyss of the pleasure and mediation, I could perform throughout day and night tirelessly. This is how Sili not only connects people from society but also connects human with spiritual entities; so, it is a means of attaining peace and harmony for living in happiness, being safe and having good luck when away from home.

Salisbury notes, "Such collective performances play a substantial role in the process of the construction of community through the practices of identity formation, sensual experiences and through social memory in which soil both gives and is given meaning and becomes a medium of expression, relying strongly on symbols about the world" 
(37). One of the most important things about performance is that they allow those who interact with them to supply some of their meaning focusing on the symbolic structure of community as well as the set of values and conventions that give meaning and a sense of identity to its members. A myriad of the symbolisms and acts as a medium of communication of ideas and tradition that Sili shows off throughout performance reflect the spectrum of diverse social rules and practices of the community. The strong symbolic connection between Sili performance and human life really enlivens the way of life and culture since their inception of practice and performance.

Regarding Sakela Sili that represents values of co-existence and harmony in the society, R. Rai enunciates:

In this convention, Sakela ranked the first, deceased forefather the second, Nakchhong the third, Silihangma and Silihangpa the fourth and common people the last for fulfilling their assigned duties. Being in structural frameworks, each and everyone in theSakela co-ordinates and co -operates for achieving common shared goals. From beginning to end of the worshiping and dancing, everyone entailing in the process actively takes part in dancing with synchronous and systematic styles and steps for making the performance successful. (Personal Interview)

Identifying and fulfilling assigned duties, each and every responsible participant attempt to maintain hierarchal base in the Sakela Sili. Conforming to rules and regulations that any tradition sets for balances the effective relationship among the members of the society. Sakela Sili, in this sense, founds the basis of social relationship, values of reconciliation, and co-operation that we frequently deal with in our reallife situation.

\section{Art and Work}

Significantly intertwining art and work in the lives of Kirant Rai, Sili represents fostering nursery, tilting, cultivating, planting and protecting crops in the Ubhauli, descending time that falls in June while in Udhauli, descending time, dance embodies harvesting work like reaping, collecting and storing collected grains into safeguard for future use (K. Rai 14). The mimesis of agrarian works as nursery fostering plantation of paddy, maize and other crops and the storage works in the performative dance and songs implies that there is close connection between arts and works. The reflections of agricultural works and others on the Sili performance forward aesthetics of art and work and vice versa. Engaging in the works either manual or mental seems to be aesthetically beautiful, and dedicating to works through performative art enlivens art and work 
reciprocal and mutual. As far as relationship between art and work relationship that Sili reflects is concerned, B. Rai reflects:

Dancing in a circle with the rhythmic music and songs evokes the sense of working in group; reworking, redancing with penchant vigor connects the artistic beauty of life with aesthetic beauty of works. The value of works for life is as equally important to as the value of art in human civilization that perpetuate for the sake of humanity and brotherhood. Sili either related to agricultural works such as Faklewa Sili, TayamaKhiyama Sili or Sili related to domestic and wild animals such as Walam Sili, Naplewa Sili etcetera reflect the way human beings act or the domestic and wild animals act and react through dance steps and styles. (Personal Interview)

The real life activities represented through Sili steps and movements bring works and arts together for producing aesthetic beauty and artistic creations. Regarding art and its relation, Dodds affirms, "The dancing steps and style has far reaching implications in which bodily movement with its particular spatial, temporal and dynamic characteristics is central to the aesthetic beauty (30). Producing intimate relationship between art and life, art and work, Sakela Sili, indeed, forms intact relation with aesthetic aspects of art, incorporating the spatial and temporal values of performance. The dance performative with rhythmic music not only bridges between our emotional heart and soothing mind but also the universal resonance of human life and the spiritual world. The artistic dance steps and the movements on the agricultural activities and social customs on the Sili closely relate to the art with labor, physical with spiritual, seen with unseen, real with imagined, life with death, and concrete with abstract.

\section{Humanity and Divinity}

Sakela Sili often correlates humanity with divinity with its artistic performance in which dancers' footsteps and lowering down hands below the chest evokes the sense of heart and humanity while hand's move above head with the harmony of music and songs refers to reverence of divine forces. The deceased ancestors remembered and worshipped during the festive also embody a part of divine world. The dance performative, relating humanity to divinity, in fact, maintains cordial relation, hierarchy and good order in between human and non-human (R. Rai, Personal Interview). The deity is believed to protect their offspring from natural calamities, diseases and evil forces, if mollified with reverence. In this regard, Sala quotes: 
Dance with musical pulsation embodying natural rhythm of life links up the inner beating of heart that pulsates through energy centers. The inherent rhythm to which we react not only instills mollifying state of mind and an expanding state of consciousness but also brings universal order to human beings. (51)

Siliperformance by the participants with vigor and vibrant musical rhythms in a symmetrical order embodies inherent echoes and rhythms of inner consciousness. On account of motion and beat that dance and music compatibly creates in the Sili performance, the natural rhythms of life and dynamics links particular to universal, consciousness to subconsciousness, mind to heart, soothing state of mind to commotional state of mind, concrete to abstract, earthly to heavenly, and terrestrial to extraterrestrial. My emic perspective on Sakela Sili that I developed through my own participation and observation on Sili performance since my childhood justifies that divine force empowers the performers to perform continuously for many days and nights with the same spirit and enthusiasm without any exhaustion. What I believe is that Sakela Sili tradition connects sense of humanity with belief of divinity since Sakela per se is believed to be an almighty deity Kirant Rai worship, dance, and revere with deep faith. With reference to Sili performance, B. Rai notes, "the vigor and vibrancy of dance and music intact with natural rhythm transform ordinary state of mind into surrealistic state of divinity. The Sili performance with drumbeat, Jyamta, a cymbal and songs connect terrestrial entity to celestial domain of consciousness." The resonance of musical rhythm and unique dance steps and bodily movement that emerged out of natural phenomena dilates horizon of human to nonhuman, and physical to spiritual.

\section{Sakela Sili andEcological Awareness}

Ever since Kirant Rai initiated organized community life, they worshipped the nature considering it as the mother well as a common abode of every living and non -living organism i. e. physical and spiritual entities. Although Kirant Rai believe in animism that claims everything regardless of living and non-living things has spirit and life, they rank the nature in the first position as creator, protector, nurturer and destroyer of all life (I. Rai). Regarding nature as essentially sacred reflection of Sakela, Nakchhong builds up an original Than, altar in steep rock structure or on the origin of water or under big a tree. In the midst of the altar, a Lung, a stone is placed upright burying beneath the ground as an idol of Sakela. Even the artificial altar for dance is built up with a big bough of chestnut, around which performers perform with differentSili to please the nature.

Sakela worship and Sili performance are closely associated with revering to nature such as stone, soil, springs, trees, plants and streams 
based on concept of 'Ecosophy' or 'Deep Ecology' proposed by Naess Arne in 1973. In this regards, Naess notes, "Ecosophy as a philosophy of ecological harmony studies how people should live with theenvironment or nature, believing in the concept that the balance between culture and nature fosters the basis of ecological harmony" (95). This is why, Sili is the product of the harmony between culture and nature, which can promote stability and harmony of the whole world. The close ties between dance and society and all things in nature signify a chain of symbiosis that develops the holistic approach. Sili as culture respects and conforms to nature and ecological aesthetic of ancient as well as modernpeople. According to B. Rai, "Kirant Rai thinks that Sakela is the amorphous form of the nature as long as the idol of Sakela made out of stone is an important part of nature." Some of the important constituents of nature such as water, stone, trees, soil and other as a whole make up the nature, which is believed to be the whole form of Sakela. Such worldview developed through constant practice and belief of Sakela Sili advances an ecological awareness on Kirant Rai community to realize the value of ecology for the sake of living and non-living beings.

The concept of ecological awareness represented through Sili performance in Kirant Rai community seems extremely valuable and panacea when the advent of modernity, urbanization, increasing aspiration of the people for a better way of life interfere the nature with greed and selfish motive. Sili such as Narowa Sili, Chhongai Sili, Jyawa Sili, WapaWama Sili that simulate movements of streams, wild birds' dance, motions and songs center on the dynamisms of the nature, that in a sense develop the conscience of nature conservation (K. Rai, 264). The mimesis of natural phenomena and humane activities on different stage of civilization, mainly of agricultural age, shape the way Kirant Rai think over ecological preservation issues and socio-cultural practices. Sili performance connects socio-cultural with natural and humanity with divinity by revering nature and divinity and maintaining the close ties between nature and culture. Turner asserts "cultural rite and shows are held in commune." (96) It means any performative arts; rites and festival must have social approval that fosters the inseparable ties among members of society. The various step-dances offer a valuable embodiment of material culture that constitutes a specific entanglement of 'nature' and 'culture', which establishes the relationship of human/non-human interactions.

\section{Sakela Sili and Earth Movement}

Sakela Sili not only represents living culture of human society, symbolic roles, human activities but also natural phenomena, occurrence and earth movement. A huge mass participation forming in circle in dance moves as the earth's shape and daily move 'axis' and yearly move 'orbit'. Led by Silihangpa and Silihangma, performers form a circle like earth 
movement and begin dancing with musical melody of Dhol, a drum, Jyamta, a cymbal and typical songs. Sakkhewalung ${ }^{4}$, an idol of a deity made out of stone, situated on the midst of the altar, is considered to be symbol of the sun in the movement of dance. Performers' first round movement is metaphorically compared with 'Axis' while the whole rounding movement of the circle is considered as yearly 'Orbit' (K. Rai 221). As a matter of fact, the movement of SakelaSili symbolically embodies the movement of the earth as an axis and an orbit. The dancers revolve around the nucleus, Sakkhewalung under a tree or artificial tree, buried to be stood for short time. Following the drumbeat music and songs, the performers sing songs and move in uniform steps, which reflect Kirant Rai's profound relationship with the mother earth and nature. The celebration is a prayer to nature for the protection from natural calamities and fatal diseases. The practices and the intimate entanglement between humans and their surroundings cement the intimacy between mother earth and human beings.

\section{Conclusion}

The paper has explored how Sakela Sili through their performance reflects the socio-cultural practices, values and ecological awareness of Kirant Rai in their day-to-day lives and vice versa. Sili represents different aspects of life, closely connecting nature with culture, man with divine, emotion with motion, real with imagined etcetera. Paruhang-Sumdi Sili, for example, demonstrates classical values of origin and historical roots, while Aatarung Sili displays haunting age. Similarly, Kharu Sili represents agricultural age while Chhekusoma Sili reflects the love affair between young boy and young girl of modern context. In this way, Salela Sili demonstrates a broader spectrum of human civilization and development through haunting age to present age. Based on the qualitative research design, particularly Interviews with Sili experts, Nakchhong, and knowledgeable elders, Focused Group Discussion, and theoretical foundational ideas of Luc Sala, Arne Naess, Dodd Sheril, and Victor Turner, the paper has interpreted Sakela Sili that establish socio-cultural behaviors and practices among Kirant Rai. Moreover, employing writer's emic and etic perspectives on Sili that imply broader scopes and more areas than one expects has made the paper valid and reliable.

The observation and self-paticipation of particular Sili and knowledge as well as perception of Sili experts, Nakchhong and other function as primary sources of data and interpretation while books, articles about Sakela Sili and other books related to theories and practices work as secondary sources of information. Cementing the ideas of culture with

\footnotetext{
${ }^{4}$ Symbolic idol of Kirant Rais' God, representing nature
} 
nature and representing socio-cultural values Kirant Rai inherently practice in their daily lives, Sakela Sili with resonance of musical rhythm and motion expand the horizon of human universe of art, culture and civilization. Worshiping and performing Sili in s circle symbolizes social solidarity and cooperation among social members, meaning that any performative arts, rites and festival must have social approval that fosters the inseparable ties among members of society. The various embodiments of material culture that constitutes a specific entanglement of nature and culture establish underlying relation in between human/non-human interactions.

\section{Works Cited}

Dodd, Sherril. Dance on Screen. New York: Palgrave Macmillan, 2004.

Naess Arne. The Shallow and Deep, Long Range Ecology movement Inquiry (16), 1973.

Puma, Prabin. Paru Sewa Mundum: Introduction, Recitation and Analysis.

Kathmandu: National Foundation for Development of Indigenous Nationalities, 2074 B.S.

Rai, Dik Bahadur. "The Impacts of Modernization on the Traditional Sakewa Sili Festival in the Rai Kirant Community of Nepal: A Case Study of the Rai Community." Unpublished M.Phil. Dissertation. Norway:Faculty of Humanities, Social Sciences and Education University of Tromso, 2012.

Rai, Bhulanath. Personal Interview. 15 June 2019.

Rai, Indra Dev. Personal Interview. 25 June 2019.

Rai, Kamal Jung Rai. Personal Interview 22 June 2019.

Rai, Kamal Jung. Sakkhewa Sili: Ek Aadhyan. Kathmandu: Dambar Bahadur Rai, 2074 B.S.

Rai, Ram Prasad. Personal Interview. 7 June 2019

Sala, Luc. Ritual the Magical Perspective. New Delhi: Nirala Publication, 2014.

Salisbury, Roderick. "Engaging with soil, past and present'. Journal of Material Culture, Vol. 17. 2012.

Shrestha, Shiv Kumar. Kiranti Folks Tales. Dhankuta: Mrs. Ganga Devi Shrestha, 2047.

Turner, Victor. The Ritual Process: Structure and anti-Structure.Chicago Il: Aldine Publishing Company, 1992. 